\title{
Estimation of Location and Activation Time of Purkinje Myocardial Junctions from Sparse and Noisy Endocardial Electrical Samples
}

\author{
Fernando Barber, Miguel Lozano, Ignacio Garcia, Rafael Sebastian* \\ CoMMLab, Universitat de Valencia, Valencia, Spain
}

\begin{abstract}
The activation of the myocardial muscle is triggered by Purkinje-myocardial junctions (PMJs), which are the terminal sites of the specialised cardiac conduction system (CCS). Obtaining the location of the PMJs and other sources of endocardial ectopic activity would be desirable for building computer models of cardiac electrophysiology and planning ablation interventions. We present a method to estimate the location and activation times of endocardial electrical sources in a $3 D$ model of the ventricles. The algorithm requires a set of discrete electrical samples on the endocardium, which can include errors in location and activation time. The estimated sources are properly placed with a location and time error in the order of the measurement error.
\end{abstract}

\section{Introduction}

In the human ventricles the electrical activation starts in the sino-atrial node, and propagates down the His bundle, the bundle braches and finally the Purkinje network (PKN). The PKN is connected to the myocardial tissue at discrete locations known as Purkinje-myocardial junctions (PMJs) that trigger the tissue depolarization from a number of remote locations [1]. The network is extensive and the location of PMJs is distributed, forming clusters or hot-spots in specific areas such as the Papillary muscle insertions. Among the most common disorders related to the cardiac conduction system (CCS) there is the left/right bundle branch block, which results in a ventricular asynchrony. There have also been described several pathologies, such as macro-reentrant tachycardia that use the CCS to sustain the arrhythmia [2, 3, 4]. Those disorders can be treated with therapies such as cardiac resynchronization therapy or radiofrequency ablation, which are known to interact with the CCS [5].

To improve those complex therapies that show low successful rates, there have been developed 3D multiscale computer models of the heart that aim at reproducing the patient disease and can be used to plan and optimize the therapy [6]. In that context, a computational model of the CCS is necessary if one wants to model its interaction with the activation sequence in health and disease. Clinical imaging techniques do not allow to recover the
PKN structure, or its PMJs since they work at the milimeter scale, which makes it impossible to differentiate the "working" myocardium from the specialized PK cells. Microscopy techniques of exvivo histological samples have been used to analyse the CCS and to describe PMJs, however, only small sections of the CCS can be analysed [7]. Therefore, typically for heart modelling, non-specific 3D CCS models have been developed using fractals, or growing algorithms among others [8].

Recently, some studies have shown techniques to inversely estimate or predict the location of PMJs from electro-anatomical maps $[9,10,11]$. The goal is to reduce the difference between simulated endocardial activation and a set of measurements obtained from the same patient's endocardium. In [9] a set of PKN are synthetically generated, and following the PMJs are moved, added or deleted to reduce the differences between measurements and simulations. In [10] the backward Eikonal problem is solved in the EAM domain to find local minima in the activation maps, which are considered the electrical sources or PMJs.

This study aims to estimate the location and activation time of all relevant endocardial sources of electrical activity from a sparse set of endocardial measurements, from now on sensor points, obtained by an electroanatomical mapping system (EAMs). Given the adequate proportion of EAM samples, the system will locate source points with a small error even in the presence of measure errors introduced by the acquisition system or the postprocessing of electrical signals.

\section{Material and Methods}

\subsection{Estimation method}

We consider the ventricular-endocardium surface as a Riemannian manifold. The activation time at the sensor points $p_{i}$ (measured using a catheter on the endocardium) will be considered as a Gaussian random variable, $\mathcal{T}_{i}$, with mean the actual activation time and a known standard deviation $\sigma$ as shown in equation 1 .

$$
\mathcal{T}_{i} \sim N\left(\tau\left(s_{j}\right)+\frac{d\left(p_{i}, s_{j}\right)}{v}, \sigma^{2}\right),
$$


where $s_{j}$ is the source (PMJ) that activated $p_{i}, \tau\left(s_{j}\right)$ is the actual activation time, $d(0$ is the geodesic distance between two points and $v$ the propagation velocity on the surface. Here we are assuming an isotropic propagation of the signal, so the geodesic distance can be calculated with a Fast Marching algorithm [11].

The sources (PMJs) are estimated in two different phases. First, given the local activation times (LAT) of all the sensor points and their geodesic distance to every point on the discretized manifold, we estimate the minimum activation time for each point in the mesh. This minimum activation time is in fact an approximation to the solution of the inverse Eikonal problem, considering the sensor points as boundary conditions. At every mesh point, if enough sensor points are compatible with this minimum activation time, the point is marked as a candidate source point (CSP). CSPs are given a score, based on the number of compatible sensor points and the evaluated errors. On a second phase, we eliminate CSPs whose associated sensor points are compatible with other CSPs with small errors. The sensor points are then associated to these other CSPs. That prevents the generation of PMJ clusters in the estimated set. This process of CSP elimination is done iteratively in the order induced by the scores, and after each iteration the scores are recalculated for all the involved CSPs. This second phase can be considered a special type of clustering of the CSPs obtained in the first phase and gives as result a minimum set of estimated PMJs that are able to explain the activation map with a small error.

\subsection{Simulation study}

To test the methodology, calibrate parameters and to compare our results with the previous works in the literature, we developed 2D scenarios based on synthetic Purkinje trees with different branch and PMJ densities. All the Purkinje trees lie on a 2D sheet of tissue of $6 \times 9 \mathrm{~cm}$ that represents the endocardium. To build the Purkinje tree structure we use an algorithm that begins with 1 primary branch (B1) or 3 primary branches (B3) and it creates new branches recursively up to a user defined level depth. In this study we used two different levels of branch recursion, depth 2 (D2) and depth 4 (D4). The algorithm and scenarios are explained in detail in [12].

For more realistic tests, we have also used 3D surface models of left ventricles reconstructed from medical imaging of real patients combined with synthetic Purkinje trees obtained following the approach in [8].

In all scenarios, the activation maps were sampled at random locations (sensor points) with increasing density to evaluate the quality of the solutions obtained with the proposed algorithm. The number of sensor points ranged from 100 to 1000 in steps of 100 points. The size of the set of sensor points is in the order of magnitude of a routinely EAM acquired in the clinic. In order to test the robustness of the algorithm, we introduced Gaussian error in the LAT of the sensor points with a standard deviation of $0.5,1.5,2.5$ and 5.0. To evaluate the solution we have propagated a new signal using the new calculated source points and we use the mean of the absolute error of the LAT in all the points of the discretized manifold. Another way to measure the quality of the solutions is to calculate how far the estimated PMJs are from the real ones. This is calculated with a Fast Marching algorithm [11] centered in each real PMJ that stops when it reaches an estimated PMJ.

\section{Results}

The results of the simulation study for 2D scenarios are summarized in Figure 3 and Figure 4. Figure 3 shows the mean of the absolute error in two scenarios: B1D2 with one primary branch and a level of recursion of 2 , and B3D4 with three primary branches and a level of recursion of 4 . The first plot is the simplest one, with only 12 PMJs, and we can see that above 200 sensor points there is no improvement in the error. The second plot in Figure 3 is a more complex one with approximately 130 PMJs and we can observe that it continuous improving with more sensor points. We can also see that the absolute error is proportional to the error in the sensor points and, in general, it is below its standard deviation. In the plot in Figure 4 we have represented the distance from the real focus (the PMJs) to the nearest estimated focus in order to measure how far the estimated focus are from the exact solution. The distance varies from $2 \mathrm{~mm}$ for the smallest deviation to $5 \mathrm{~mm}$ for a deviation of $5.0 \mathrm{~ms}$. It must be noted that this error in the estimation of the position is not only due to the error of the sensor or the algorithm, but also to the fact that some PMJs are not effective, i.e. they have no influence at all in the LAT of the domain, and so they are impossible to detect.

In Figure 2 we can see a particular example of the B3D2 scenario in which we included a standard deviation error in the sensor of $1.5 \mathrm{~ms}$ in the LAT. The mean of the absolute error in the LAT obtained for this scenario was $0.98 \mathrm{~ms}$. In this scenario there are several PMJs which are not effective.

In Figure 5 we have a 3D scenario with 1.000 sensor points with a standard deviation in the sensor error of 0.5 ms. The mean of the absolute error in the whole mesh was $0.65 \mathrm{~ms}$. 


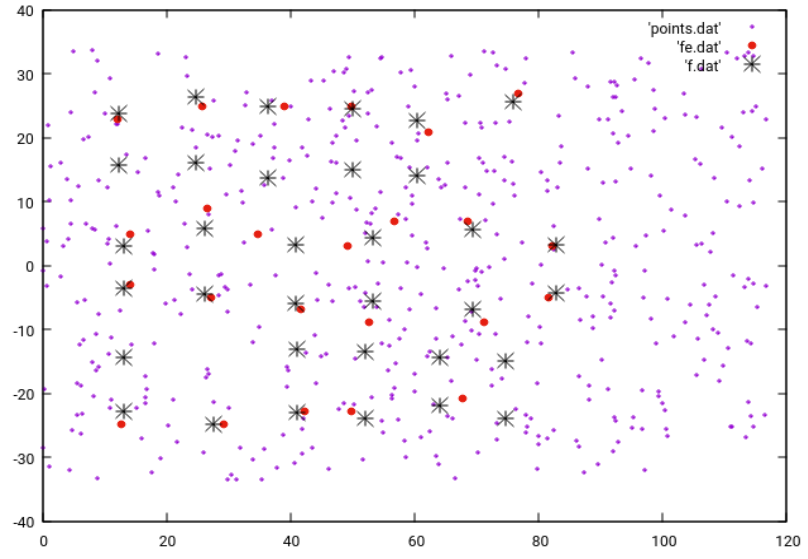

Figure 2: 2D scenario B3D2 with random set of sensor points (small dots), real sources (stars) and location of estimated source points (red spheres). Standard deviation in the sensor error of $1.5 \mathrm{~ms}$. Mean error obtained for the scenario: $0.98 \mathrm{~ms}$.
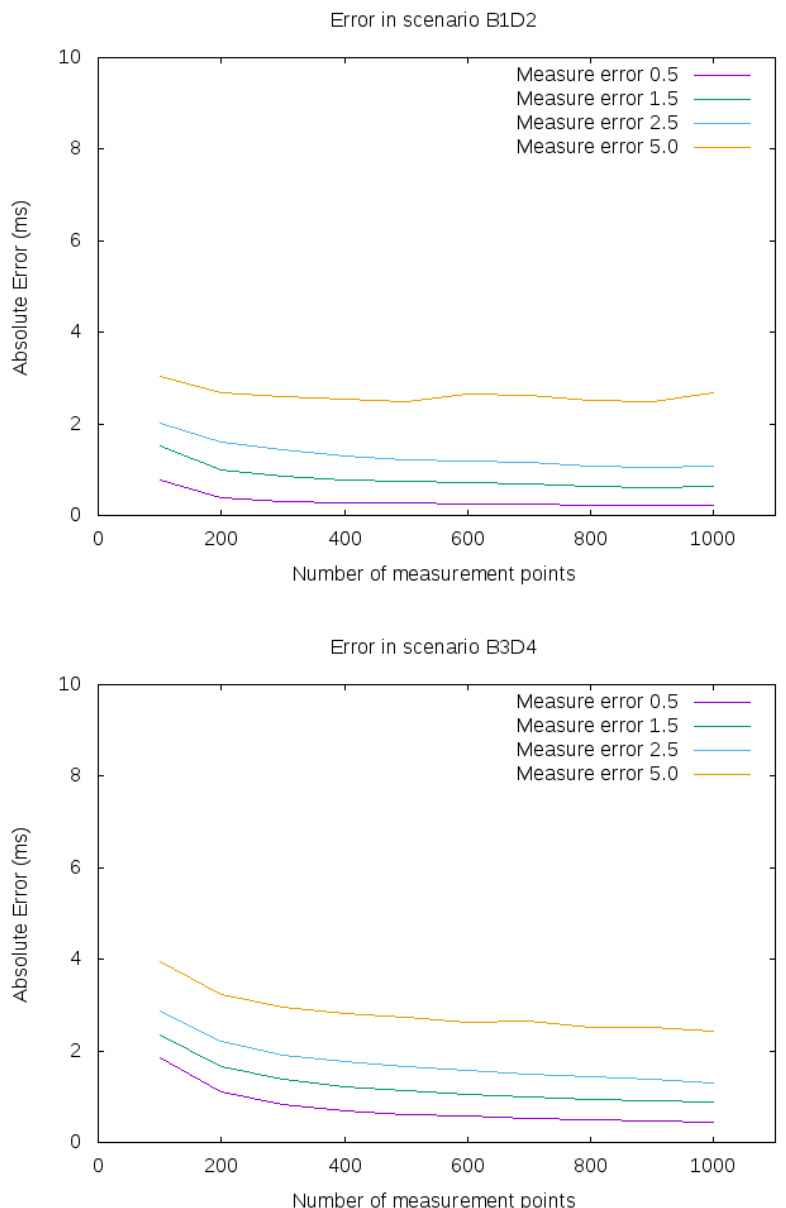

Figure 3: Absolute error in scenarios B1D2 and B3D4 with a standard deviation error in the sensor of $0.5,1.5$, 2.5 and 5.0 milliseconds.

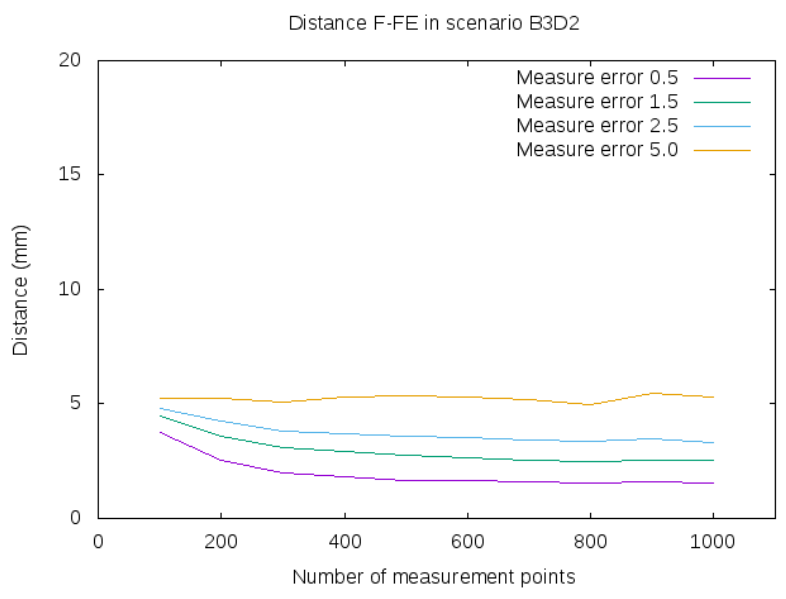

Figure 4: Distance from a real focus (PMJ) to the nearest estimated focus in scenario B3D2 with a standard deviation error in the sensor of $0.5,1.5,2.5$ and 5.0 milliseconds.

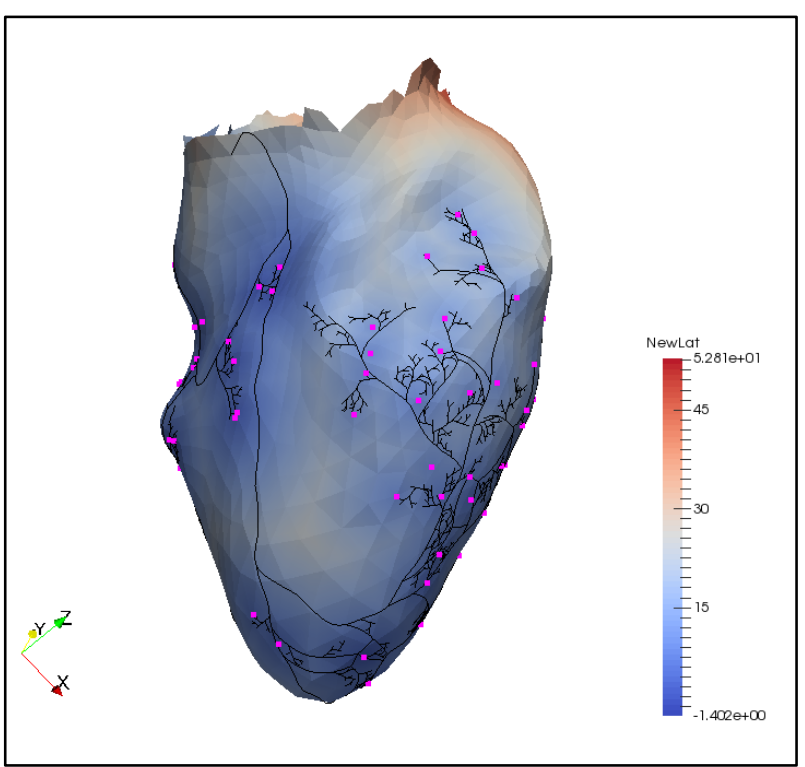

Figure 5: Detected PMJs (purple dots) for a 3D scenario with 1.000 sensor points with a standard deviation in the sensor error of $0.5 \mathrm{~ms}$. The mean of the absolute error in the whole mesh was $0.65 \mathrm{~ms}$.

\section{Discussion and conclusions}

We have presented an algorithm that can be used to obtain the location of sources of electrical activity in the ventricles even in the presence of error in the sensor points. Using the information from all the measurement points in a 3D domain, the algorithm converges to those endocardial areas where PMJs are found. This allows the use of the algorithm for obtaining a set of real activation 
points for the ventricles and hence produce more realistic simulations of the heart. In cases in which a large number of PMJs is clustered in a region, it cannot differentiate each of them individually and as a results produces a few PMJs that summarize the whole group. PMJs within clusters have none or a very local impact in the overall activation map, since most of them are non-effective nodes. The signal emitted by cluster inner PMJs is quickly masked by the PMJs at the border of the tree and they are mainly non-effective PMJs. Differently to other similar methods, the algorithm is designed to obtain the location of PMJs, and not only for reproducing the electrical activation sequence.

One of the drawbacks of the method is that it requires a minimum number of sensor points, in the order of 1000 , to obtain a sufficiently large number of PMJs which can be time consuming for an electrophysiologist. Although new systems can acquire larger number of measurements, they are not widely available yet.

\section{Acknowledgements}

This study is supported by the e3DTorso project (TIN2014-59932-JIN) from the Spanish Ministerio de Ciencia y Competitividad and FEDER funds.

\section{References}

[1] D. Durrer, R. Van Dam, G. Freud, M.J. Janse, F.L. Meijler, and R.C. Arzbaecher. "Total excitation of the isolated human heart.," Circulation, vol. 41, pp. 899-912, 1970.

[2] E. Behradfar, A. Nygren, and E.J. Vigmond . "The role of Purkinjemyocardial coupling during ventricular arrhythmia: a modeling study.," PLOS ONE, Vol. 9, pp. e88000, 2014

[3] E. Ben Caref, M. Boutjdir, H. D. Himel, and N. ElSherif, "Role of subendocardial Purkinje network in triggering torsade de pointes arrhythmia in experimental long QT syndrome.," Europace, vol. 10, no. 10, pp. 1218-23, Oct. 2008.

[4] T. Sakata, H. Tanner, T. Stuber, and E. Delacrétaz, "His-Purkinje system re-entry in patients with clustering ventricular tachycardia episodes.," Europace, 2008, vol. 10, no. 3, pp. 289-93.

[5] D. Romero, R. Sebastian, B. Bijnens, V. Zimmerman, P.M. Boyle, E.J. Vigmond, and A.F. Frangi, "Effects of the Purkinje system and cardiac geometry on biventricular pacing: a model study.," Annals of Biomedical Engineering., 2010, vol. 39, no. 4, pp. 13881398.

[6] H.J. Arevalo, F. Vadakkumpadan, E. Guallar, A. Jebb, P. Malamas, K. C. Wu and N. A. Trayanova. "Arrhythmia risk stratification of patients after myocardial infarction using personalized heart models.," Nature Communications., 2016, vol. 7, pp. 11437.

[7] V. Garcia-Bustos, R. Sebastian, M. Izquierdo, P. Molina, F.J. Chorro, and A. Ruiz-Sauri, "A quantitative structural and morphometric analysis of the Purkinje network and the Purkinje-myocardial junctions in pig hearts," Journal of Anatomy, 2017, vol. 230, no. 5, pp. 664-678.

[8] R. Sebastian and V. Zimmerman, "Characterization and modeling of the peripheral cardiac conduction system," IEEE Med. Imaging, 2013, vol. 32, no. 1, pp. 45-55.

[9] R. Cárdenes, R. Sebastian, D. Soto-Iglesias, A. Berruezo, and O. Camara, "Estimation of Purkinje trees from electro-anatomical mapping of the left ventricle using minimal cost geodesics.", Medical Image Analysis 2015, vol. 24, no. 1, pp 52-62.

[10] S. Palamara, C. Vergara, E. Faggiano, F. Nobile, "An effective algorithm for the generation of patient-specific purkinje networks in computational electrocardiology", Journal of Computational Physics 2015, vol. 283, pp. 495-517.

[11] .J. A. Sethian, "Fast Marching Methods", SIAM Review, vol. 41 issue 2, pp. 199-235, June 1999.

[12] F. Barber, M. Lozano, I. García-Fernández, R. Sebastian, "Automatic Location of Sources of Electrical Activation from Electroanatomical Maps", Computing in Cardiology 2016, vol. 43, pp 445-448.

Address for correspondence:

Rafael Sebastian

Computational Multiscale Simulation Lab (CoMMLab), Universitat de Valencia. Escuela Técnica Superior de Ingenierías. Av. de la Universidad s/n, 46100 - Valencia (Spain). rafael.sebastian@uv.es 